\title{
The cross sectional anatomy of ventricular septal defects: a reappraisal
}

\author{
E J BAKER, * M P LEUNG, $\dagger$ R H ANDERSON, D R FISCHER, J R ZUBERBUHLER \\ From the Division of Cardiology, Children's Hospital, Pittsburgh, Pennsylvania, USA
}

SUMMARY. The cross sectional echocardiographic description of holes in the ventricular septum has been unsatisfactory, chiefly because there are so many classifications of this defect. The accurate description of the anatomy of individual defects, from cross sectional images, is more important than attempts to fit them into a preconceived classification. One hundred specimens of hearts with a ventricular septal defect were reviewed to identify those features that are of value in the interpretation of cross sectional images. Three groups of defect were identified: those which abutted the central fibrous body, those with a margin partly formed by an area of fibrous continuity between the leaflets of the aortic and pulmonary valves, and those with entirely muscular margins. Each group had features that were readily discernible in cross section. Other features of the defects seen in the cross sectional images identified defects that opened between the two ventricular inlets, defects that opened between the two subarterial outlets, and those that extended solely into the trabecular septum. The criteria for describing the anatomy of defects were established in hearts with normal connections, but they were found to be equally applicable in hearts with discordant atrioventricular connection, discordant ventriculoarterial connection, common arterial trunk, and double outlet from the morphologically right ventricle. Cross sectional imaging, by echocardiography and potentially by other techniques, provided a uniquely detailed and precise description of the morphology of ventricular septal defects.

The understanding of the morphology of holes between the ventricles has been complicated by the plethora of existing and proposed schemes of classification. Several cross sectional echocardiographic studies of the anatomy of ventricular septal defects have been published. ${ }^{1-3}$ In each, the authors interpreted the echocardiographic images by reference to an established classification. Despite these reports, or perhaps because of them, uncertainties remain about the interpretation of cross sectional images of ventricular septal defects. We have, therefore, restudied the morbid anatomy of ventricular septal defects to identify those morphological features that are important in the interpretation of cross sectional images.

Requests for reprints to Dr E J Baker, Department of Paediatric Cardiology, Guy's Hospital, London SE1 9RT.

\#Present address: Department of Paediatric Cardiology, Guy's Hospital, London SE1 9RT.

†Present address: Grantham Hospital, University of Hong Kong.

Accepted for publication 21 September 1987

\section{Patients and method}

We examined a representative series of specimens with a ventricular septal defect from the heart museum of the Children's Hospital of Pittsburgh. Hearts with an atrioventricular septal defect, pulmonary or aortic atresia, or a univentricular atrioventricular connection were excluded. The margins of each defect were carefully inspected to identify the features that were amenable to cross sectional imaging. Where necessary, hearts were sectioned to simulate echocardiographic planes and to clarify the cross sectional appearances. After the anatomical study, cross sectional echocardiograms from patients with ventricular septal defects studied at the Children's Hospital, Pittsburgh were selected to illustrate some of the important morphological features.

\section{Results}

In all, 100 hearts were examined (table). Fifty two had a ventricular septal defect in the setting of usual (concordant) chamber connections. In addition, we 
Table $A$ list of hearts examined with the category of ventricular septal defect

\begin{tabular}{lr}
\hline Connection & No \\
\hline Normal connections: & 42 \\
Perimembranous & 8 \\
Muscular & 2 \\
Doubly committed and juxta-arterial & 4 \\
Complete transposition: & 9 \\
Perimembranous & 1 \\
Muscular & \\
Doubly committed and juxta-arterial & 2 \\
Congenitally corrected transposition: & 1 \\
Perimembranous & 1 \\
Doubly committed and juxta-arterial & 14 \\
Both doubly committed and perimembranous & 1 \\
Double outlet of the right ventricle: & 4 \\
Perimembranous & \\
Muscular & 8 \\
Doubly committed and juxta-arterial & 3 \\
Common arterial trunk & \\
Perimembranous & \\
Muscular right ventricular margin &
\end{tabular}

examined 18 hearts with ventricular septal defect and a discordant ventriculoarterial connection-14 of these had a concordant atrioventricular connection and four a discordant atrioventricular connection. A common arterial trunk was present in 11 hearts, all

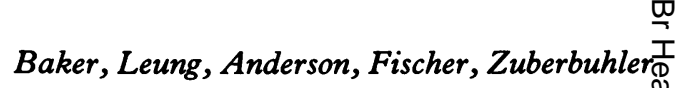
with a concordant atrioventricular connection. $A$ final group of 19 hearts was studied with double outlet from the morphologically right ventricle, again $\frac{\vec{s}}{\overrightarrow{3}}$ in the setting of a concordant atrioventricular con- $\overline{0}$ nection. Some of the hearts in this series have beeno described in previous studies. ${ }^{4-6}$

\section{HEARTS WITH CONCORDANT}

ATRIOVENTRICULAR AND VENTRICULOARTERIALC CONNECTIONS

Borders of the defect: holes abutting the central fibrous body

In hearts with otherwise normal anatomy, the centralo fibrous body could be identified as the area of $c$ continuity between the tricuspid, aortic, and mitraliw valves. When a hole in the septum abuts the centrale fibrous body, it is also, therefore, immediately? adjacent to the fibrous structures supporting theleaflets of the aortic valve (fig 1a). In that sense, it is "subaortic". ${ }^{2}$ To an extent, this is a confusing term. Often, there is a considerable length of fibrous tissue between the edge of the defect and the origin of the $e_{\infty}^{\circ}$ leaflets of the aortic valve (fig 1b). Furthermore, many observers would consider a defect to be more응

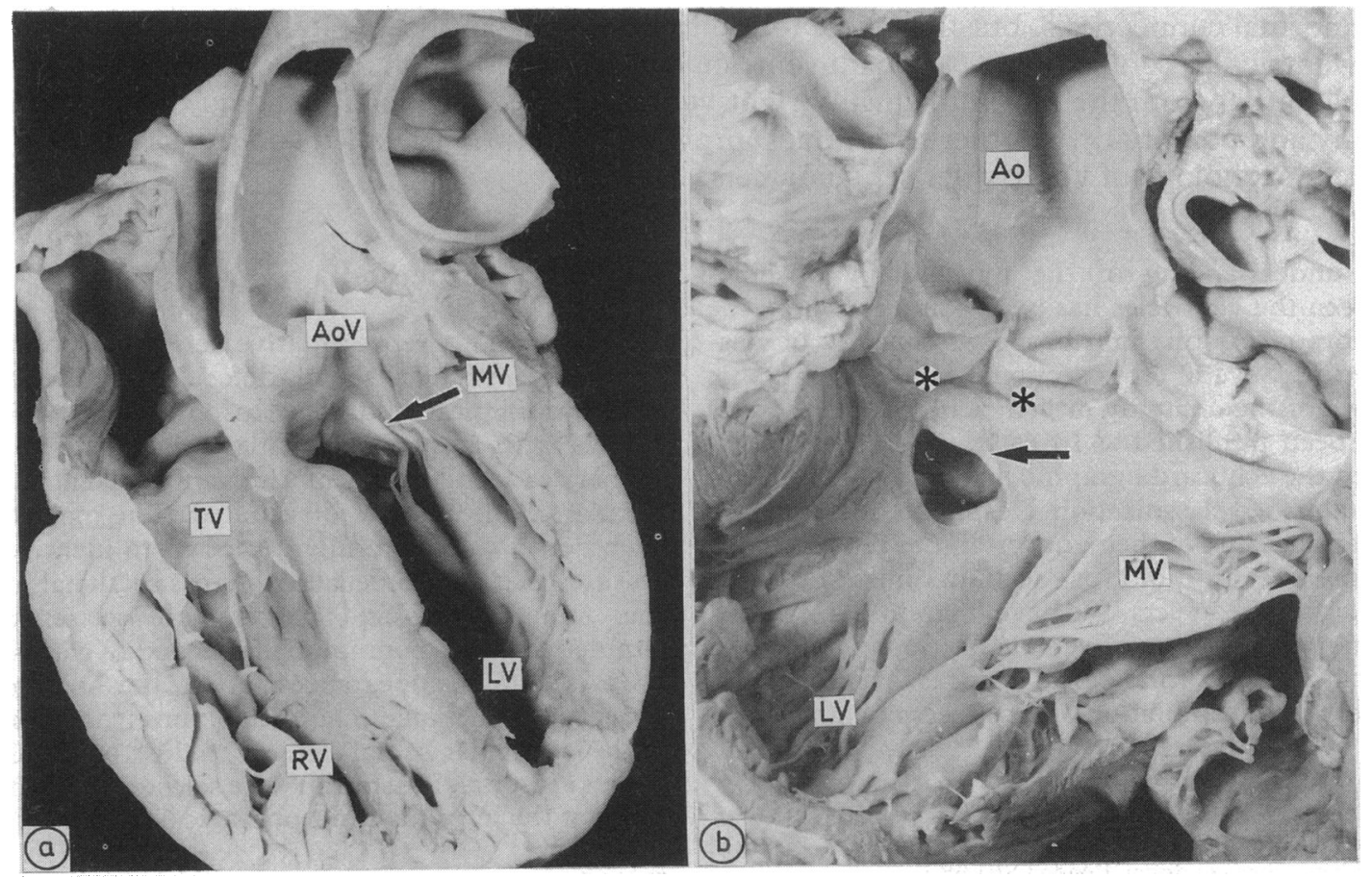

Fig 1 (a) This simulated four chamber cut, incorporating the aortic (Ao) root, shows how the cardinal feature of the defect we choose to call perimembranous is fibrous continuity between the tricuspid (TV), aortic (AoV), and mitral (MV) valves in the roof of the defect. In some circumstances, as shown in a view from the left ventricle (b), a substantial ridge of fibrous tissue (arrowed) may hang below the attachments of the leaflets of the aortic valve (asterisks). $R V$, right ventricle. 
obviously "subaortic" when there is overriding of the valve orifice as, for example, in the tetralogy of Fallot. There is, none the less, a need to describe specifically those defects bordered superiorly by the fibrous tissue supporting the aortic root. A major component of this tissue is the atrioventricular membranous septum and the defects are disposed around this fibrous partition. For this reason, and despite the reservations expressed by others, we continue to describe this group of defects as perimembranous. ${ }^{7}$

Perimembranous defects were the most common in this series. The openings of some of them extended into the inlet, outlet, or trabecular zones of the ventricles. Small defects could be judged morphologically to be opening into the right ventricular inlet or outlet by virtue of their relation to the medial papillary muscle. Defects above the papillary muscle have been termed "outlet", and those beneath it "inlet", while trabecular extension is indicated by insertion of the papillary muscles at the apical margin of the defect. ${ }^{7}$ This morphological distinction was difficult to make in the hearts in which the medial papillary muscle was multiple or ill defined. Furthermore, this relation seemed unlikely to be identified by cross sectional imaging. Thus for imaging purposes we considered it inappropriate to use the terms "inlet", "outlet", and "trabecular" for the description of small perimembranous defects. Large perimembranous defects extending posteriorly to open into the right ventricular inlet formed a well defined group, as did those large defects that extended anteriorly to open into the subpulmonary right ventricular outflow tract. In contrast, large perimembranous "trabecular" defects did not form a distinct group. Indeed, they had no morphological features that distinguished them from perimembranous defects extending to open into both the outlet and inlet of the right ventricle. This group was, therefore, described as "confluent".

\section{Holes bordered by fibrous continuity between the leaflet of the aortic and pulmonary valves}

We identified a further discrete group of defects in which at one margin there was direct fibrous continuity between the leaflets of the aortic and pulmonary valves (fig 2). Such defects always opened between the two subarterial outlets. They involved an abnormality of septation of this area, as do all outlet defects. These defects have previously been called "supracristal". Our preference is to describe them as doubly

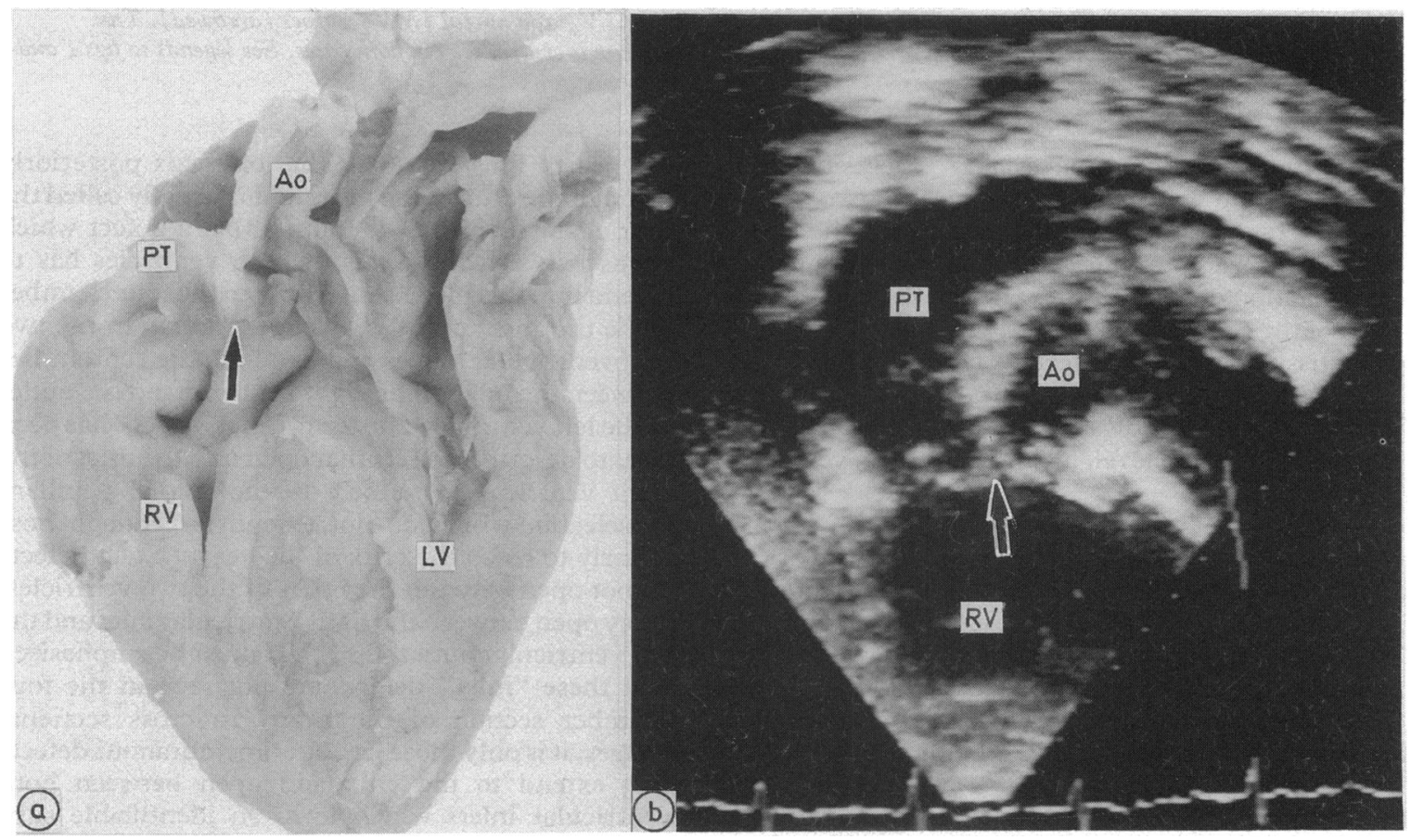

Fig 2 These illustrations show the cardinal features of the defect we choose to call doubly committed and juxta-arterial. The anatomical specimen is sectioned in a simulated parasternal long axis plane. The defect is roofed by fibrous continuity between the arterial valves (arrowed). The echocardiogram is an oblique subcostal cut and shows both great arteries (pulmonary trunk $(P T)$ and aorta (Ao)) apparently above the right ventricle (RV). There is clearly fibrous continuity between the arterial valves (arrowed). LV, left ventricle. 

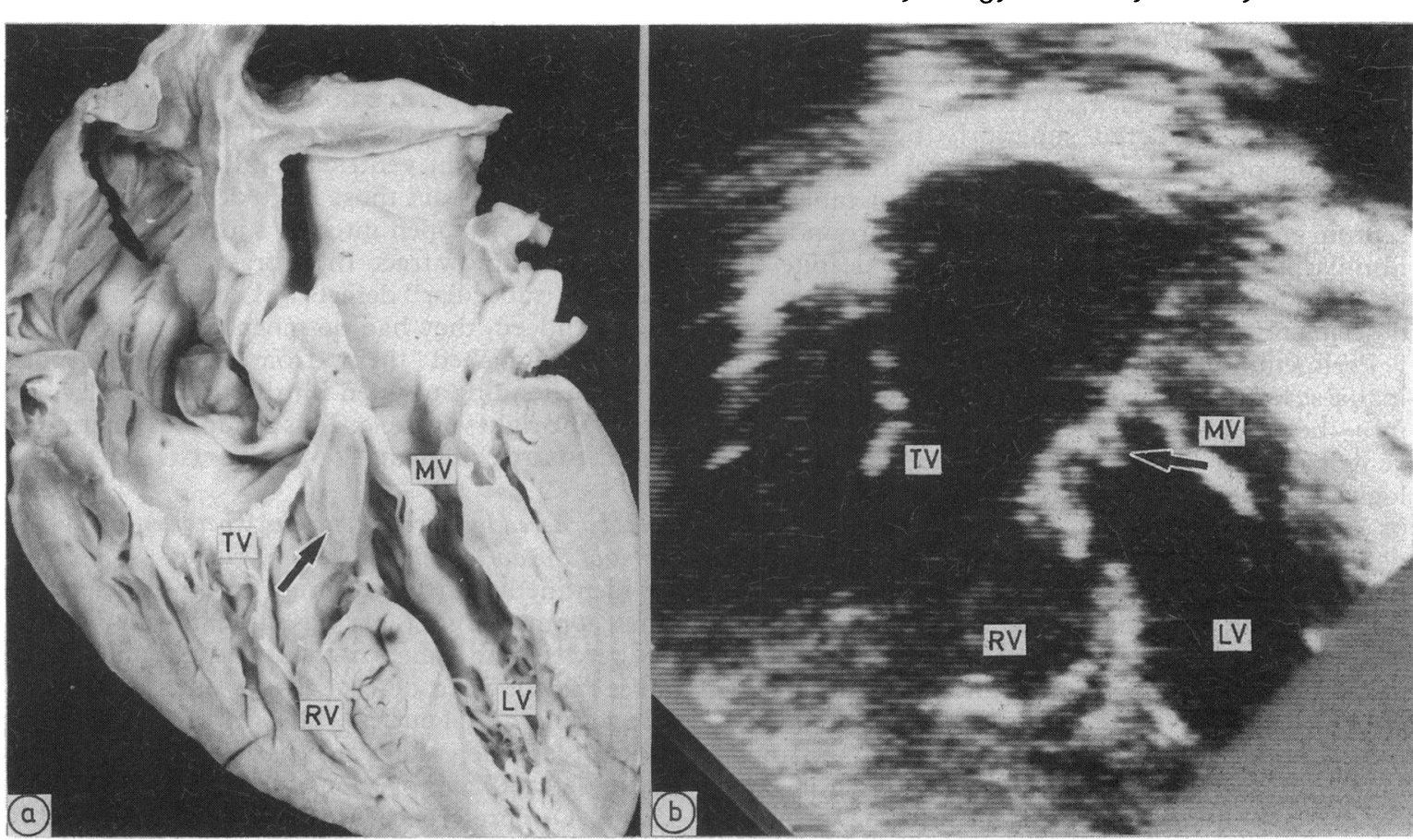

Fig 3 These figures show defects that are not perimembranous opening between the ventricular inlets. A bar of muscle separates the defect from the area of continuity between the tricuspid (TV) and mitral (MV) valves (arrowed). The offsetting of the insertion of the two atrioventricular valves to the septum is clearly seen in both cases. See legends to figs 1 and -2 for abbreviations.

committed and juxta-arterial. ${ }^{78}$ Occasionally a fibrous raphe was present between the two arterial valves. In cross section the leaflets of the valves usually appeared to be at the same level, but careful examination of some of these hearts showed that, for part of the extent of the defect, the valves were offset. The tissue between the leaflets was composed of the arterial wall of a sinus of Valsalva. At some point along the defect, however, the leaflets were always found at the same level, albeit over only a very small area.

\section{Holes with entirely muscular margins}

All ventricular septal defects which were not perimembranous and were not doubly committed and juxta-arterial had a margin that was entirely muscular (fig 3). Such defects could be further described according to their position within the muscular septum and their relation to the arterial valves.

Description of the position of holes in the ventricular septum: defects opening between the ventricular inlets The "inlet" septum, the septum that separates the inlet of the right ventricle from the inlet of the left ventricle, can only be identified in a single plane of the heart. This passes in the long axis posteriorly through the crux of the heart and is usually called the four chamber plane.' It follows that a defect which? opens between the inlets of both ventricles has to extend into this plane. Anteriorly to the four chamber plane the aortic valve is "wedged" between the two atrioventricular valves and, so, here the septum lies 3 . between the inlet of the right ventricle and the outlet of the left ventricle. The term "inlet defect". has been used to describe defects that open into the inlet of theo right ventricle posteriorly to the medial papillary muscle but which do not extend far enough pos teriorly to reach the crux of the heart. ${ }^{7}$ Such defects do not open between the inlets of the two ventricles. They open between the right ventricular inlet and the left ventricular outlet (fig 1a). It must be emphasised that these "inlet" defects are not seen in the fouro chamber section of the heart.' In cross sectionab images; it is only those large perimembranous defect 5 ह that extend to the crux and open between both? ventricular inlets which form an identifiable subgroup. In our series perimembranous defects extend $\frac{0}{P}$ ing to the crux were not common. We judged than in 12 hearts the defect belonged to this category Continuity between tricuspid and mitral valves formed one margin of these defects and normab 

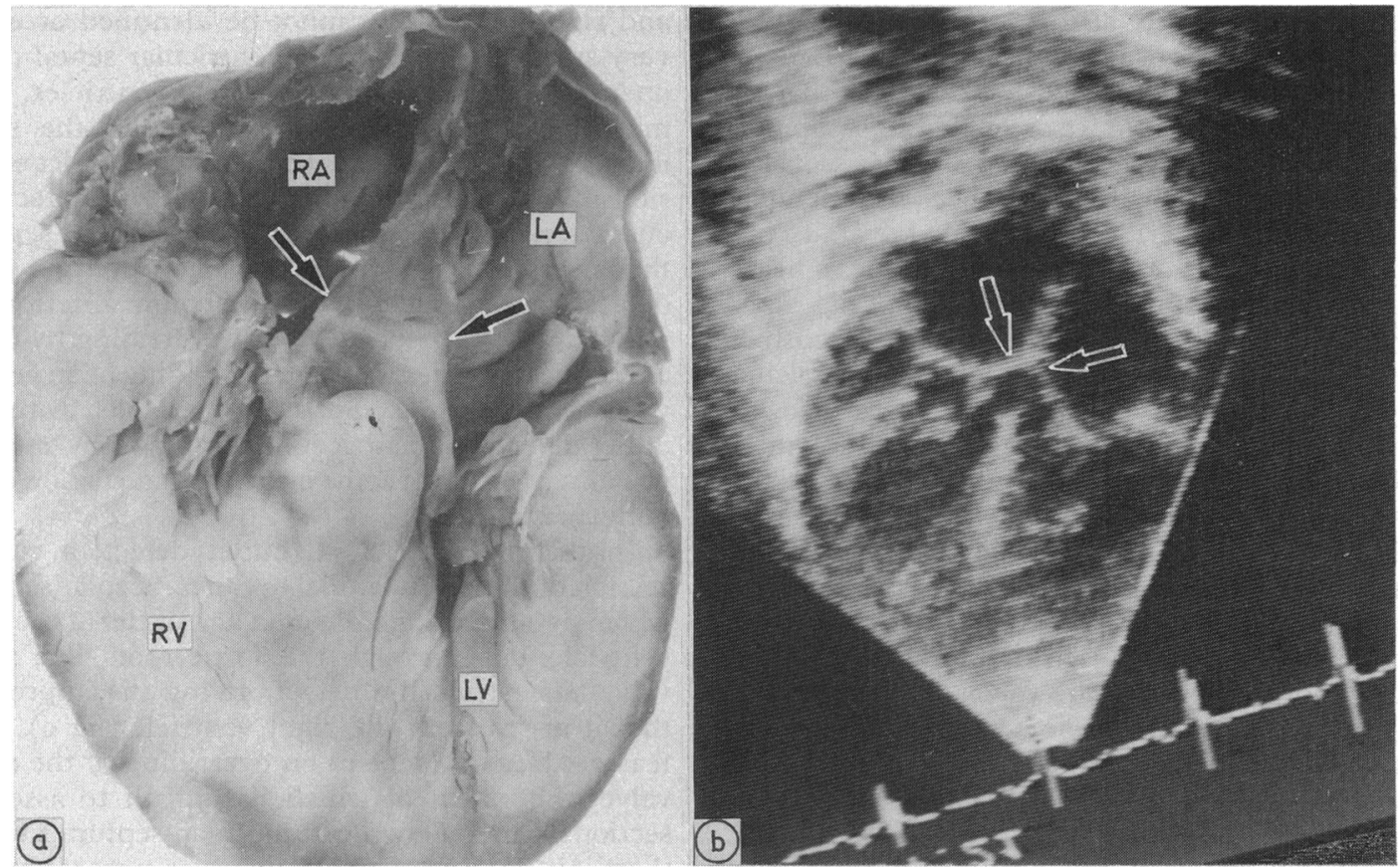

Fig 4 These sections of a perimembranous defect (not from the same patient) show that the defect extends to the crux, opening between the inlets of the two ventricles. The mitral and tricuspid valves (arrowed) are inserted into the septum at more or less the same level. $R A$, right atrium; $L A$, left atrium; $R V$, right ventricle; $L V$, left ventricle.

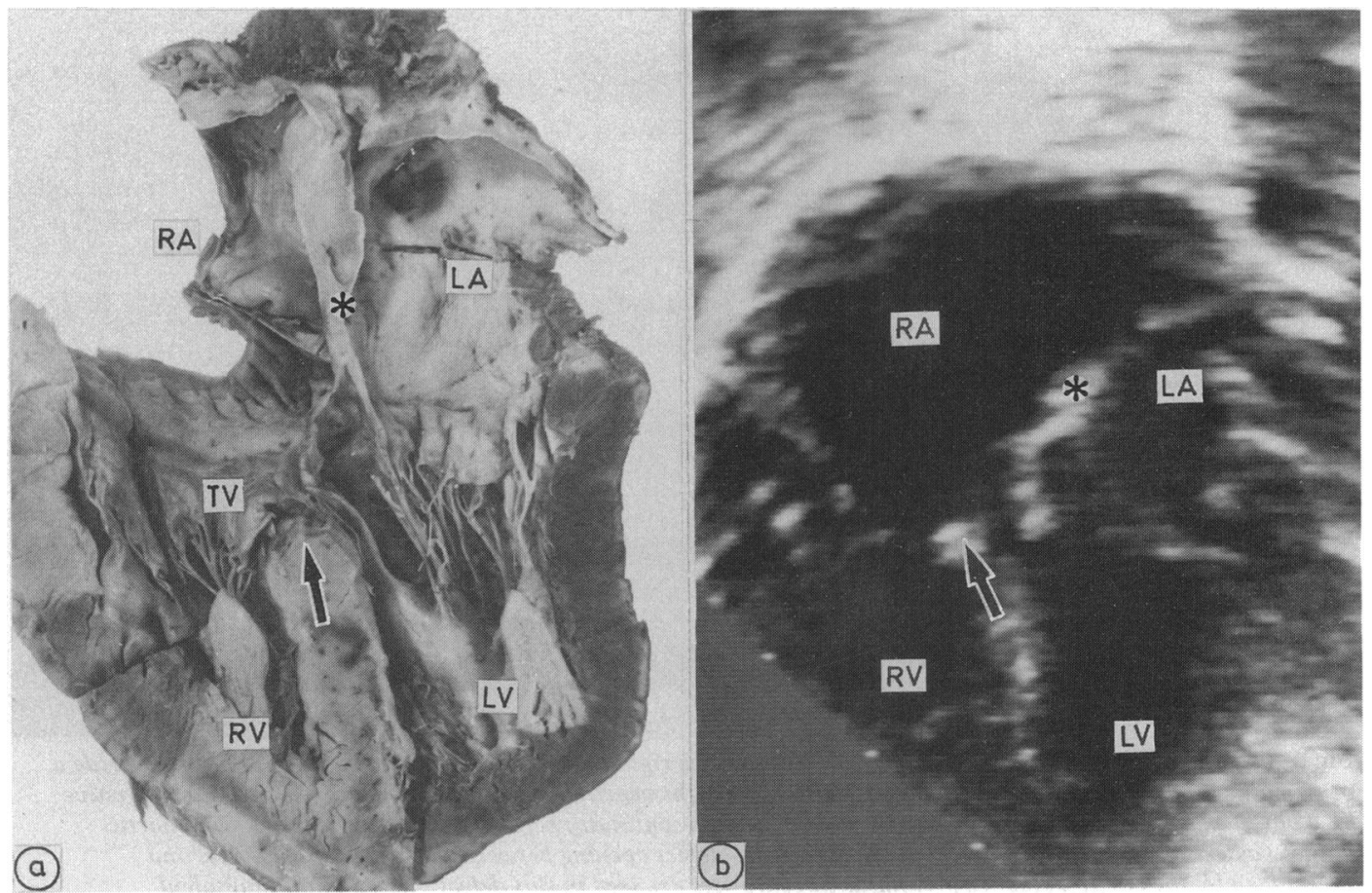

Fig 5 These sections (not from the same patient) show the malalignment between the atrial (asterisk) and ventricular (arrowed) septal structures that is the hallmark of the inlet defect associated with straddling and overriding of the tricuspid valve (TV). See legends to figs 1 and 2 for abbreviations. 
offsetting of the two atrioventricular valves was absent (fig 4). In one heart in this group, the tricuspid valve overrode and straddled the defect so that the inferoseptal commissure was attached to a papillary muscle within the left ventricle. This was associated with considerable malalignment between the atrial septum and the posterior component of the muscular ventricular septum (fig 5). None of these hearts showed the morphological stigmata of atrioventricular septal defects ("endocardial cushion defects") and none had trileaflet left atrioventricular valves. They are appropriately described as perimembranous defects opening between the inlets of both ventricles-they should not be called "atrioventricular canal defects".

Muscular defects opening between the ventricular inlets also extended into the four chamber plane. They were, in contrast, separated from the atrioventricular valves by a muscle bundle and the normal offsetting of insertion of the valves into the septum was preserved (fig 3). In one heart with a muscular inlet defect, the defect straddled the tricuspid valve.

Holes between the subarterial outlets

The relation between the aortic and pulmonary valves in normal hearts is such that a true "outlet septum", a septum separating the outflows of the left and right ventricles, cannot be identified or else is very small. If, therefore, a ventricular septal defect opens between the outlets of both ventricles, there must be a fundamental difference in the septal morphology of this area. In this series, outlet extension of a perimembranous or muscular defect was characterised by the presence of a true outlet septum, the lower border of which formed one margin of the defect (fig 6). Deviation of this septum anteriorly or posteriorly was associated with overriding by either of the great arteries. Indeed, overriding of an arterial valve was a specific, but not invariable, feature of outlet defects. Subaortic ${ }^{5}$ or subpulmonary ${ }^{6}$ stenosis was also a feature of some hearts with deviation of the outlet septum.

In the presence of an outlet defect, a coronal section of the heart parallel to the trabecular septum, which passes through the inlet and outlet of the right ventricle, cuts through the aortic root. The aortic valve sits astride the defect, giving the impression that it arises from the right ventricle (fig 6). This feature does not depend on overriding of the aortic valve, the extent of which is difficult to assess in sections parallel to the trabecular septum. Where there is little or no override, however, a more anterior section will demonstrate the ventriculoinfundibular fold separating the ascending aorta from the right

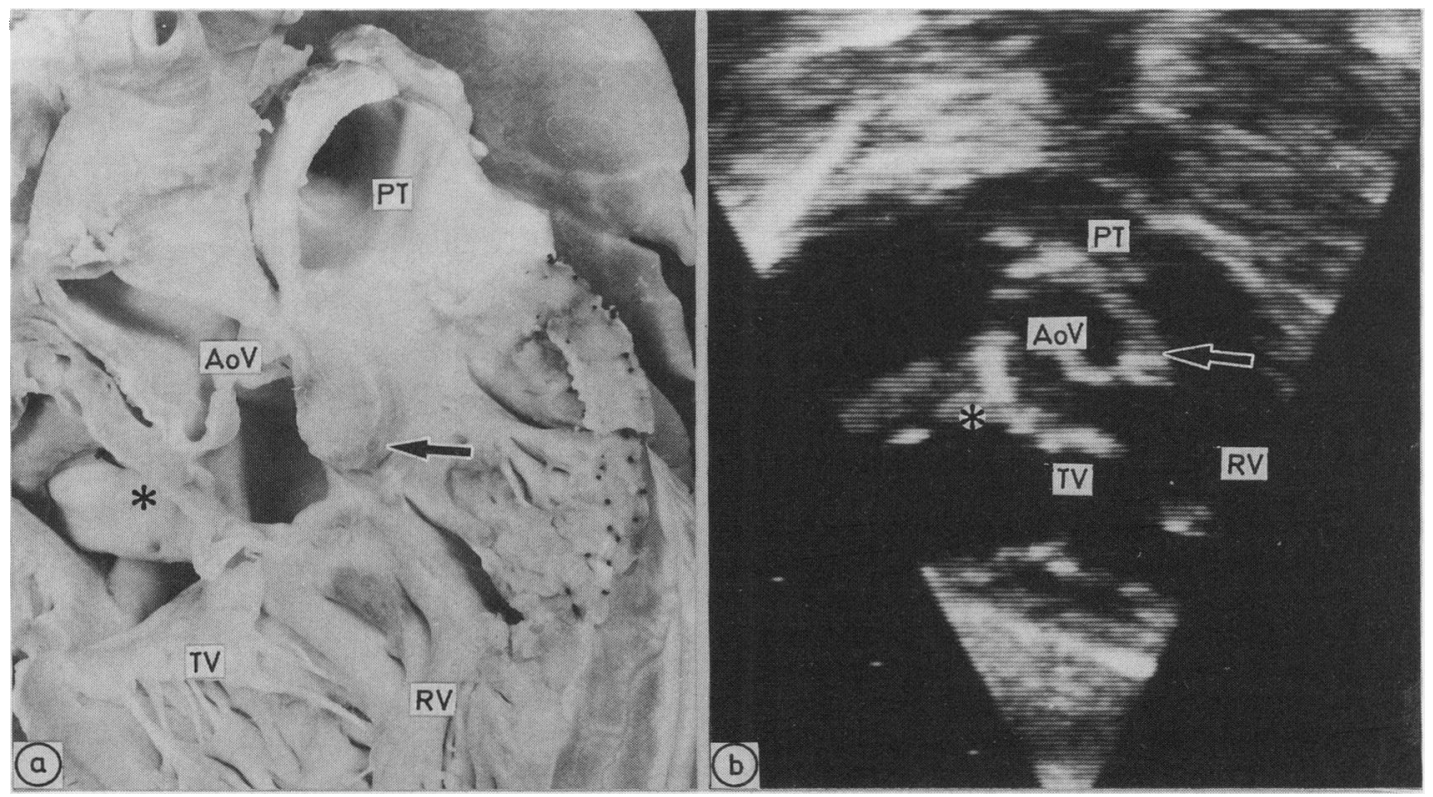

Fig 6 (a) A simulated oblique subcostal cut through the right ventricle. The aortic valve (AoV) sits astride a ventricular septal defect and appears to arise from the right ventricle. It is separated from the pulmonary valve by a bar of muscle, the outlet septum (arrowed). There is continuity between the tricuspid (TV) and aortic valves (asterisk). This is therefore a perimembranous defect opening between the ventricular outlets and overridden by the aortic valve. (b) Similar appearances are seen in this defect viewed in an equivalent echocardiographic plane. $R V$, right ventricle; $P T$, pulmonary trunk. 


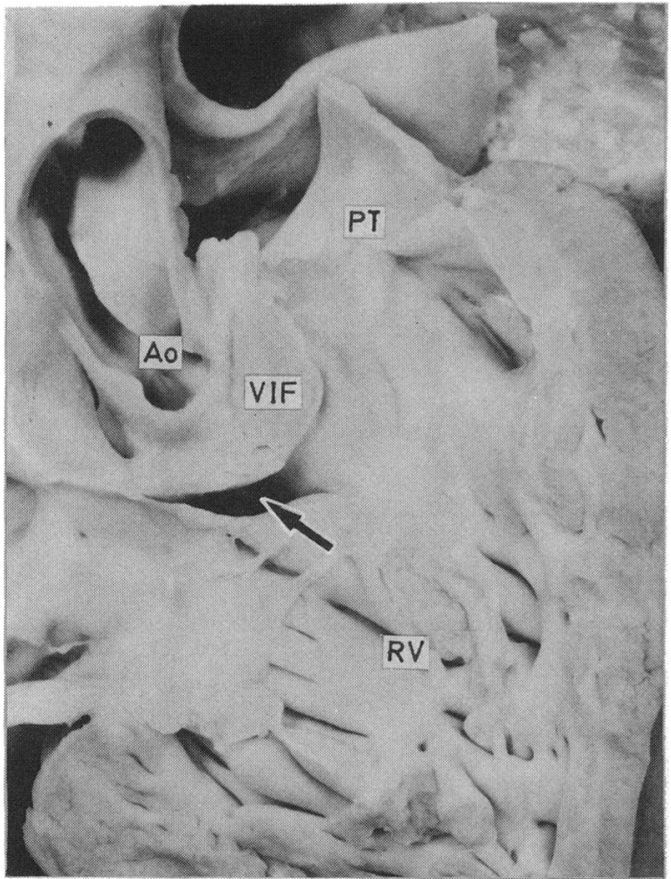

Fig 7 A simulated oblique subcostal cut in a heart with a perimembranous defect (arrowed) opening between the ventricular outlets with no override. The ventriculoinfundibular fold (VIF) separates the aorta (Ao) from the right ventricle (RV). $P T$, pulmonary trunk.

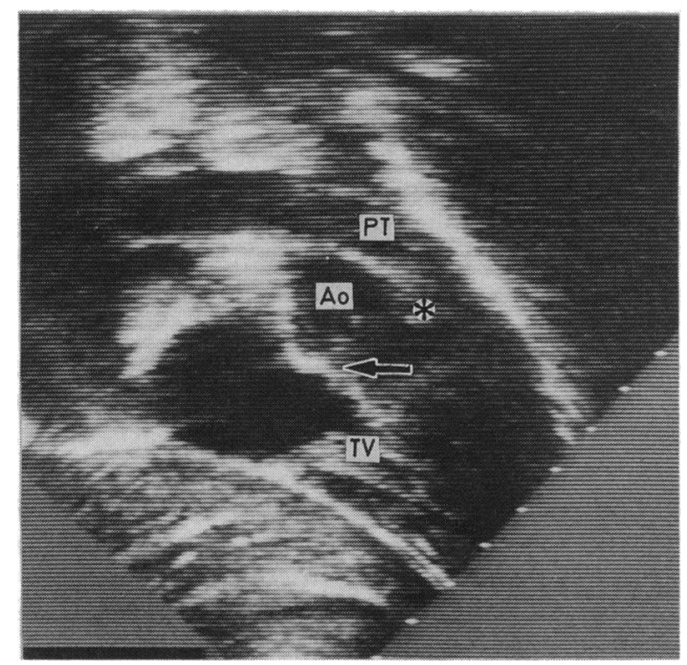

Fig 8 An oblique subcostal cut in a patient with a defect that was both perimembranous and doubly committed and juxta-arterial. There is fibrous continuity between both the tricuspid (TV) and aortic valves (arrowed) and the aortic and pulmonary valves (asterisk). See legends to earlier figures for abbreviations. extremely valuable in the definition of outlet defects. The absence of a muscle bar between the aortic and tricuspid valves indicated that the defect was perimembranous (fig 6 ). If there was no muscle bar between the aortic and pulmonary valves (that is no outlet septum and therefore an incomplete subpulmonary infundibulum) the defect was doubly committed and juxtaarterial (fig 8). A muscle bar was present at both sites in muscular outlet defects (fig 9) and absent from both sites in defects that were both perimembranous and doubly committed.

\section{HEARTS WITH COMPLETE TRANSPOSITION}

The morphological features of defects in hearts with a discordant ventriculoarterial connection were essentially similar to those already described. There was one important difference. The two great arteries usually arise side by side from the heart and therefore there can be a true outlet septum in the absence of a ventricular septal defect. Nevertheless, muscular and perimembranous defects that extend so as to open into the two subarterial outlets were still characterised by demonstration of the outlet septum at one margin. In fact, the most common defect in this group of hearts was a muscular defect at the border between the outlet septum and the trabecular septum (fig 10). This was usually anterior to the septomarginal trabeculation, but occasionally was more posterior, in one case extending to become perimembranous. Deviation of the outlet septum anteriorly into the right ventricular outflow tract or posteriorly into the left ventricular outflow tract was a common accompanying feature.

It is usually the pulmonary valve, not the aortic valve, that is continuous with the central fibrous body in these hearts. Perimembranous defects were, therefore, identified by pulmonary to tricuspid continuity at one margin. In one heart in this group there was a complete muscular infundibulum beneath both arterial valves. Neither arterial valve was attached to the central fibrous body.

\section{HEARTS WITH DISCORDANT ATRIOVENTRICULAR CONNECTION}

The morphological features of the defects in these hearts divided them into two groups. In all four hearts studied, the hole opened between the subarterial outlets. In two cases the outlet septum was absent and a muscle bundle separated the defect from the central fibrous body (fig 11a). These defects were doubly committed and juxta-arterial. In the other two hearts, the area of fibrous continuity between the pulmonary and tricuspid valves formed part of the morphologically right ventricular margin of the defects, which were therefore perimembranous (fig $11 \mathrm{~b})$. In both cases there was rightward deviation of 


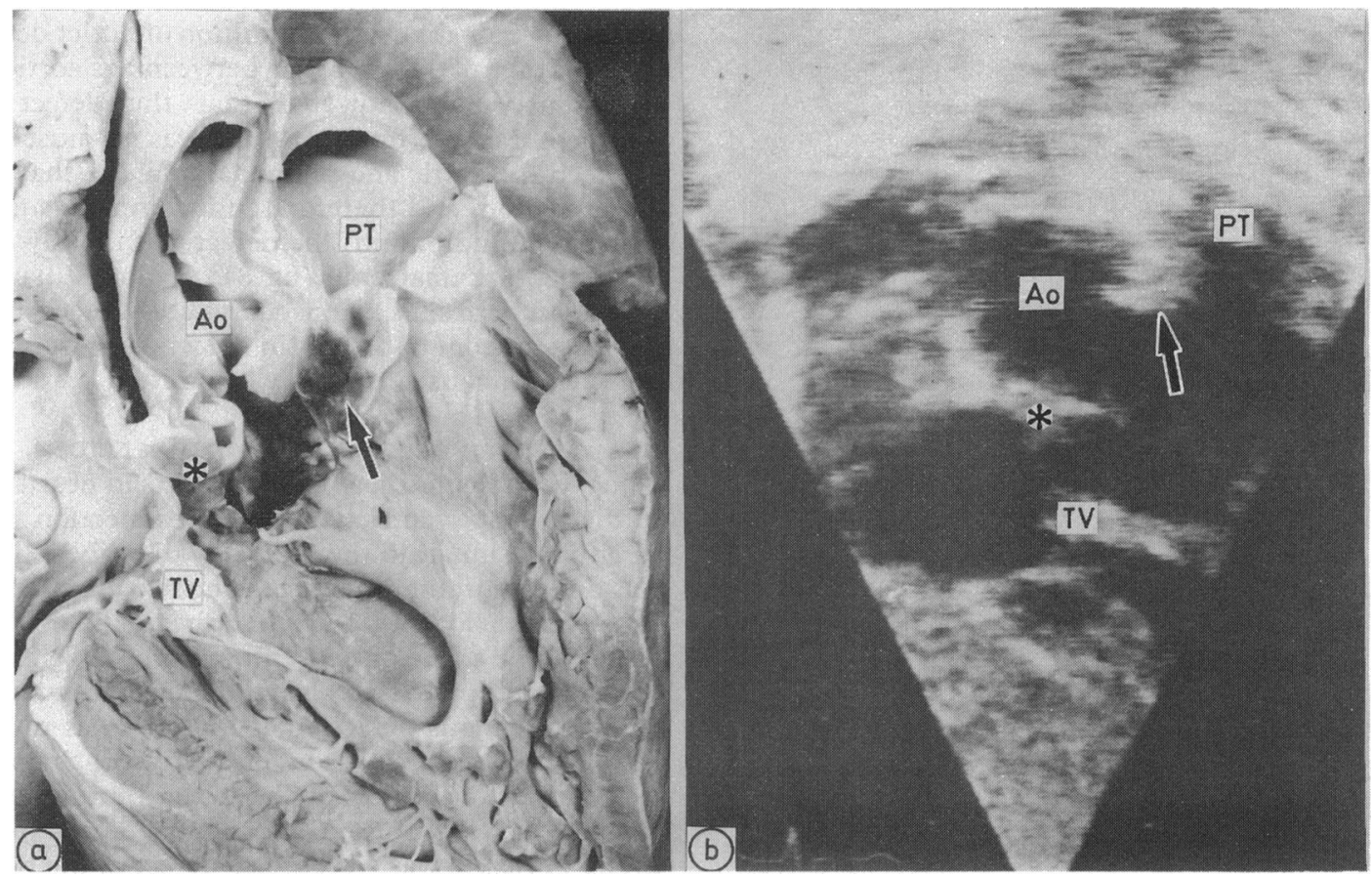

Fig 9 These two sections (not from the same patient) show a muscular outlet defect. (a) A simulated oblique subcostal cut. (b) An equivalent echocardiographic section. In both cases a muscle bar, the outlet septum (arrowed), separates the arterial valves and another muscle bar (asterisk) separates the aortic and tricuspid valves. See legends to earlier figures for abbreviations.

the outlet septum and subpulmonary obstruction. The abnormal connection in hearts with a discordant atrioventricular connection did not present any problems in the identification of the borders of the defects in cross section. The important morphological features could be readily described by the criteria already outlined.

\section{HEARTS WITH DOUBLE OUTLET FROM THE RIGHT} VENTRICLE

Many of the hearts with this connection had ventricular septal defects opening into both subarterial outlets, the essential morphology of which was not different from that of the other outlet defects that have been described. These hearts were arbitrarily classed as double outlet because the connection of the overriding aortic or pulmonary valve was judged to be greater than $50 \%$ to the right ventricle. ${ }^{10}$ If an outlet septum was present it was completely within the right ventricle and formed part of the right ventricular margin of the defect. If the central fibrous body also formed part of the right ventricular border, the defect was described as perimembranous. If not, it was described as muscular. Absence of the outlet septum was a feature of doubly committed juxtaarterial defects. In such a case, the fibrous continuity between the arterial valves formed part of the right ventricular margin of the defect. In all these defects, the left ventricular margin was partly made up by the area of fibrous continuity between an arterial valve and the mitral valve. Inferiorly, the crest of the $\vec{\varnothing}$ trabecular septum formed the common left and right ventricular border of the defect. In one heart in $\bar{\sigma}$ this group, with a perimembranous defect and an overriding pulmonary valve, the mitral valve strad- $\delta$ dled the defect. The anterolateral commissure was

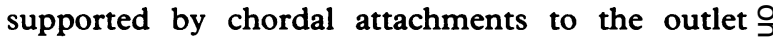
septum.

A subgroup of hearts with double outlet right ventricle had a complete muscular infundibulum $N$ beneath both arterial valves. In these hearts, neither arterial valve was continuous with the central fibrous 0 body and, therefore, in no section of the heart was $\tilde{\omega}$ there fibrous continuity between an arterial and atrioventricular valve. A perimembranous defect was $\varrho$ identified solely by the demonstration of continuity $\mathbb{D}$ of the tricuspid and mitral valves at one margin of $\stackrel{+}{+}$ the defect. With this morphological arrangement, 0 neither great artery was immediately adjacent to a perimembranous defect. In these hearts, the relation of the defect, be it perimembranous or muscular, to $\stackrel{\AA}{\AA}$ either arterial valve was variable and was not predictable from its morphology. 


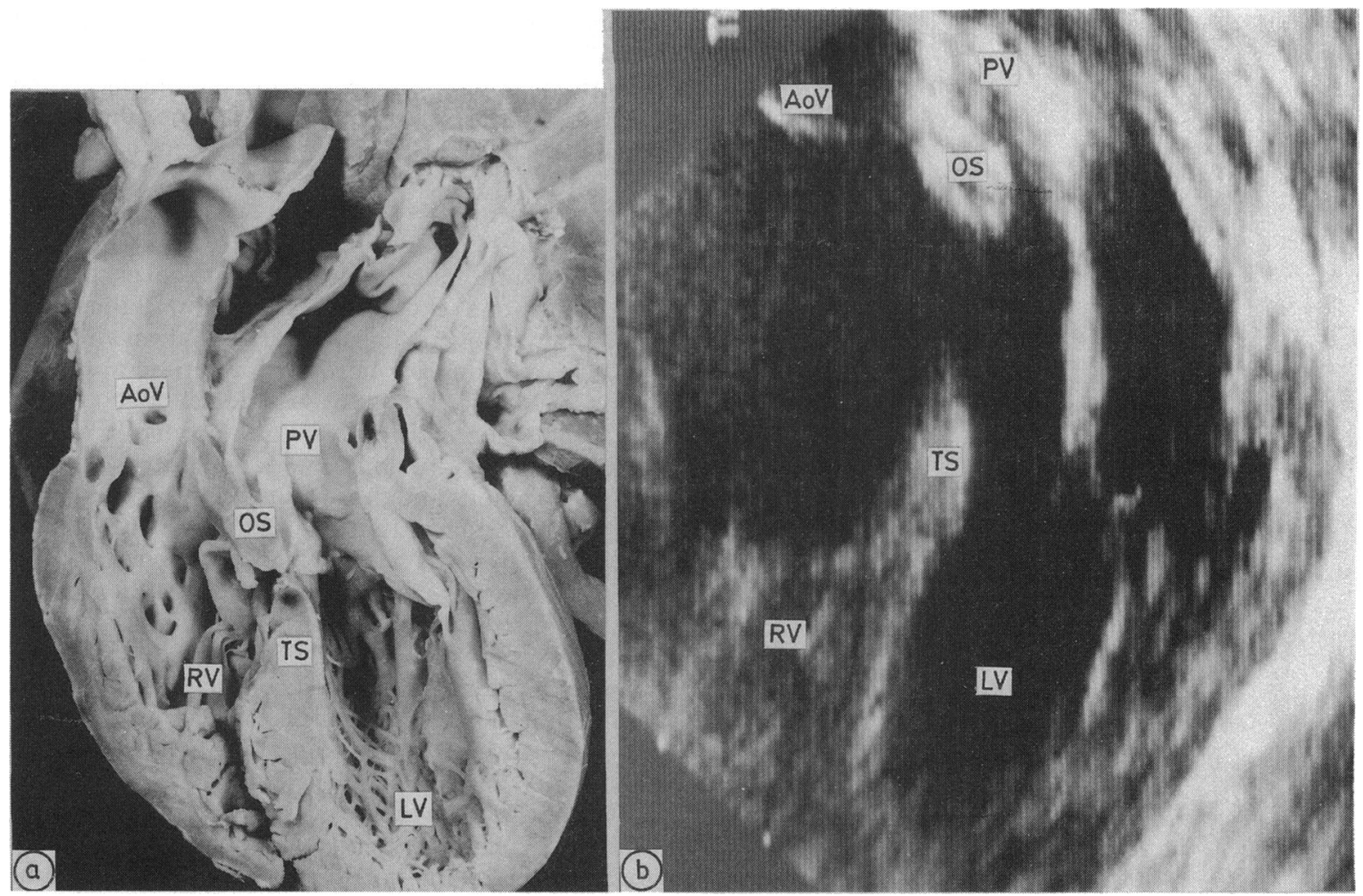

Fig 10 (a) A simulated parasternal long axis cut in a heart with complete transposition. A small ventricular septal defect is seen between the lower border of the outlet septum (OS) and the trabecular septum (TS). (b). An equivalent echocardiographic plane in another patient with a similar defect. In this case the defect is much larger and there is posterior deviation of the outlet septum causing subpulmonary obstruction. AoV, aortic valve; PV, pulmonary valve; $R V$, right ventricle; $L V$, left ventricle.

HEARTS WITH A COMMON ARTERIAL TRUNK

The ventricular septal defect in these hearts was morphologically similar to the doubly committed and juxta-arterial defect. In over half the hearts examined, the right ventricular margin of the defect included the central fibrous body (fig 12a). In other words, the defect was, in addition, perimembranous. The central fibrous body was identified as the area of continuity between the truncal and tricuspid valves. In the remaining hearts, the right ventricular margin of the defect was entirely muscular (fig 12b). In all the hearts examined, the left ventricular margin was partly made up by the area of fibrous continuity between the truncal valve and the mitral valve. In other words, there was no complete subtruncal infundibulum. Another feature of these defects was the degree of commitment of the truncal valve to the two ventricles. This was variable, but in the majority of cases the valve had more extensive right ventricular margins.

\section{Discussion}

Fully to describe a hole between the ventricles requires knowledge of several disparate features of its morphology. One of the reasons for disagreements about classification is that different groups have chosen different features as their basis. ${ }^{11}$ All the features are important and all, therefore, should ideally be described. A description based upon cross sectional imaging techniques requires that the essential.morphological features of each type of defect are identified or excluded. It does not matter in which imaging plane each morphological feature is sought. No feature should be considered to be specific to a single plane. The criteria we have identified can be applied in the interpretation of cross sectional echocardiography, but equally they are applicable to newer imaging methods such as magnetic resonance or computerised tomographic imaging, in which different imaging planes may be used. Our study has 


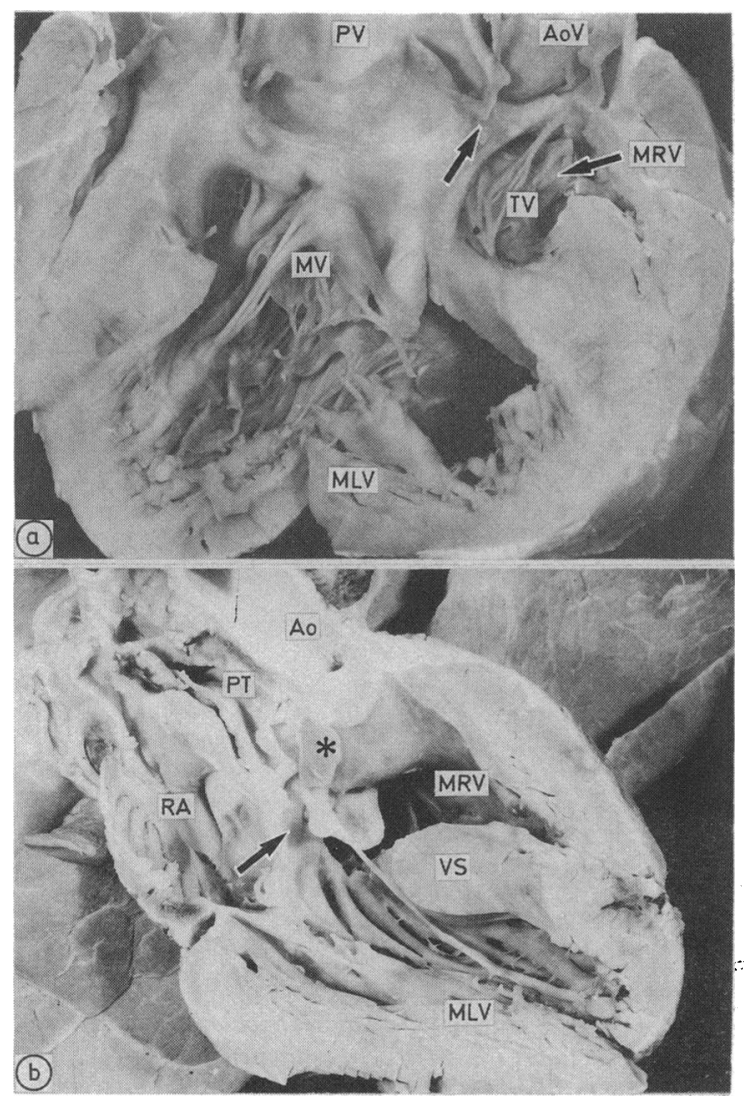

Fig 11 These sections are both from hearts with congenitally corrected transposition (discordant atrioventricular and ventriculoarterial connections). The upper section (a), in a four chamber plane, shows a defect that is both perimembranous and doubly committed and juxta-arterial. The defect extends from continuity between the atrioventricular valves (MV and TV) and reaches to continuity between the arterial valves ( $P V$ and AoV (arrowed)). MLV, morphologically left ventricle; $M R V$, morphologically right ventricle. The lower section $(b)$ is an oblique subcostal cut showing a perimembranous defect, roofed by pulmonary-mitral valve continuity (arrowed), with posterior deviation of the outlet septum (asterisk) giving subpulmonary obstruction. The left sided tricuspid valve has been replaced. Ao, aorta; $P T$, pulmonary trunk; $R A$, right atrium; VS, ventricular septum.

convinced us that different types of defect cannot adequately be distinguished exclusively by the imaging plane in which they appear. This is unsatisfactory even in hearts with normal relations. In hearts with abnormal relations, such a rationale is useless. In contrast, describing defects according to morphological features specific to each type is independent of the relations of the cardiac chambers. Others have largely tried to interpret cross sectional
Baker, Leung, Anderson, Fischer, Zuberbuhler echocardiographic images in the light of pre-existing classifications, ${ }^{12} 13$ without taking into account the limitations inherent in cross sectional imaging. The great strength of these images is the fine anatomical detail that can be identified at the margins of defects.

\section{DEFECTS BORDERING THE CENTRAL FIBROUS} BODY

In hearts with concordant atrioventricular connec- $\vec{\circ}$ tion, with one exception (see below), the non- $\overrightarrow{\vec{\omega}}$ branching atrioventricular bundle is always related $\stackrel{\omega}{\omega}$ to the posteroinferior border of a perimembranous defect. With other types of ventricular septal defect of the conduction axis follows its normal course. ${ }^{14} \underset{\omega}{\omega}$ Making the distinction between defects that are $\dot{\omega}$ perimembranous and those that are not is, therefore, 0

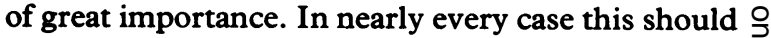
be possible by cross sectional imaging. The criterion for diagnosing a defect as being perimembranous is the demonstration at one of its margins of fibrous côntinuity between the tricuspid valve and either an arterial valve or the mitral valve. To exclude perimembranous extension of a defect, the areas of $\infty$ fibrous continuity between the atrioventricular valves and the arterial and tricuspid valves must be shown not to form one of its borders. All perimembranous defects in hearts with normal connections are adjacent to the aortic valve, but not all "subaortic" defects are perimembranous. Demonstration that a defect is "subaortic" is not, therefore, sufficient to identify it as perimembranous. Continuity of the tricuspid and aortic valves at one margin of the defect must be established. In hearts with discordant ventriculoarterial connection, perimembranous defects are generally related to the pulmonary valve and so continuity between the pulmonary and tricuspid valves must be sought. No great artery is attached to the central fibrous body in hearts with a bilateral infundibulum. This feature can be demonstrated in cross sectional imaging by the absence of fibrous continuity between either arterial valve and either atrioventricular valve. Here, it is fibrous continuity between the two atrioventricular valves at one margin that is the sole marker for a perimembranous defect. Such a defect is not immediately adjacent to either arterial valve. Classifying such defects as "subaortic", "subpulmonary", or "non-committed" does not imply anything about the morphology of the defect but rather describes the extent to which the hole and the arterial valve are adjacent. ${ }^{15}$

When the remnant of muscle between the valves is very small a muscular defect could be mistaken for a perimembranous defect. The resolution of the imaging technique would be the critical factor. Failure of resolution would be unusual, however, and 


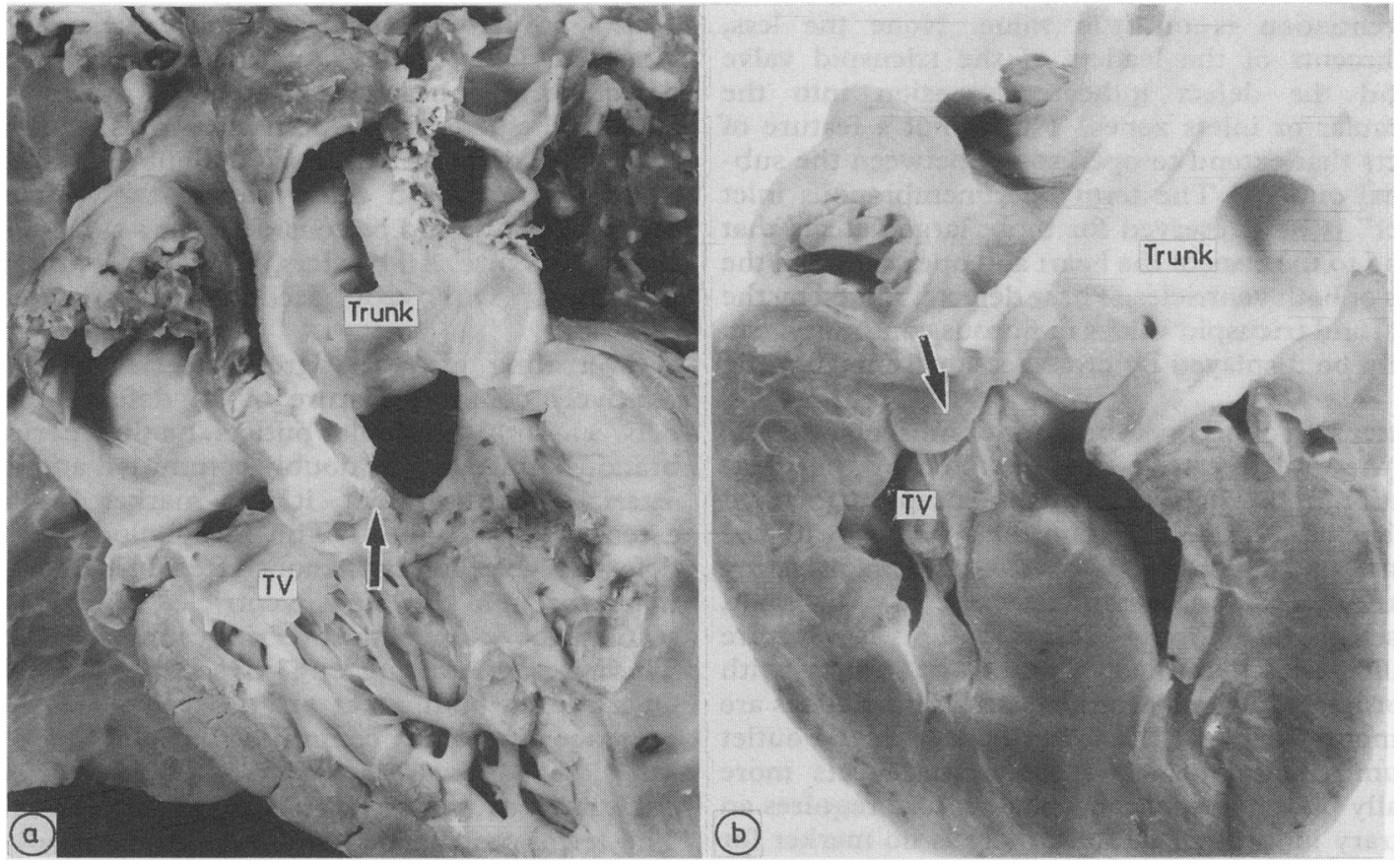

Fig 12 These two sections of hearts with a common arterial trunk show (a) a perimembranous defect, with tricuspid (TV) truncal valve continuity (arrowed), and (b) a defect with a muscular posteroinferior rim (arrowed). This is the ventriculoinfundibular fold separating the truncal and tricuspid valves.

in all the hearts we examined the muscle bordering the defect that separated the valves was substantial enough to be identified without ambiguity by current imaging techniques.

\section{HOLES BORDERED BY CONTINUITY BETWEEN THE AORTIC AND PULMONARY VALVES}

Doubly committed juxta-arterial defects are identified in cross sectional images by the presence at their superior margin of direct fibrous continuity between the leaflets of the aortic and pulmonary valves. A fibrous raphe between the two valves may be impossible to distinguish from a hypoplastic outlet septum. There is a group of defects, therefore, which will be difficult to place within the appropriate category. This distinction is, fortunately, not of great clinical importance. The two arterial valves are usually demonstrated to be at the same level, although this is not a critical feature of the doubly committed defect. Where there is a fibrous raphe or where the arterial sinus forms a margin of the defect ${ }^{16}$ the valves may truly be offset.

The defect associated with a common arterial trunk is morphologically similar to the doubly committed defect. Identifying such a defect is part of the overall diagnosis of the cardiac anomaly. It may, in addition, be perimembranous, in which case there will be continuity between the truncal valve and tricuspid valve at the right ventricular margin of the defect.

\section{MUSCULAR DEFECTS}

A defect can only be said to have entirely muscular margins when it has been shown to be neither perimembranous nor doubly committed and juxtaarterial. Demonstrating that a defect has two muscular margins in a single cross sectional plane does not prove that muscle encompasses all its margins. If there is an overriding arterial valve, it is the right ventricular margin of the defect that is important in making the distinction between it being perimembranous or muscular. If there is a muscle bundle separating the tricuspid valve from the arterial valve, or valves, connected to the right ventricle, then it is this which protects the atrioventricular conduction axis. $^{14}$

\section{POSITION OF DEFECTS WITHIN THE SEPTUM}

Cross sectional imaging can identify small perimembranous defects but cannot show whether they open into the inlet, outlet, or trabecular parts of the right ventricle. The morphological criterion for such distinction, the relation of the defect to the medial papillary muscle, cannot be used. In any case, this 
differentiation is of little value. None the less, attachments of the leaflets of the tricuspid valve around the defect indicate extension into the trabecular or inlets zones. This is not a feature of defects that extend to open solely between the subarterial outles:" The term "perimembranous inlet defect" is best reserved for those large defects that extend to the crux of the heart and open between the inlets of both ventricles. These defects, roofed by the mitral and tricuspid valves in fibrous continuity, can readily be displayed by cross sectional imaging (fig 7).

Extension of perimembranous or muscular defects into the outlets can be demonstrated by the identification, at one margin, of an outlet septum, defined as a bar of muscle separating the space beneath the two arterial valves. As we have seen, demonstrating that the defect is adjacent to the aortic valve does not, in itself, indicate outlet extension. This is a feature of all perimembranous defects. In hearts with discordant ventriculoarterial connection, defects are commonly found at the lower border of the outlet septum. Distinguishing these from defects more apically placed in the trabecular septum requires an arbitrary judgement because there is no marker for the level of the junction of the outlet and trabecular septum.

Morphologically, perimembranous defects that extend into both the inlet and outlet parts of the right ventricle can be termed "confluent". Cross sectional imaging permits the identification of a very large defect that extends both to the crux and into the subarterial outlets. But a confluent defect that does not extend to the crux cannot be distinguished from a perimembranous outlet defect.

Muscular defects that do not open into the outlets may be identified as opening between the ventricular inlets if they are seen in the septum immediately beneath both atrioventricular valves and are separated from them by a bar of muscle. Other muscular defects can be described as trabecular. These defects can be further characterised by the distance they are from the apex in long axis sections. They can be multiple and have a tortuous course and hence, if small, may be difficult to detect in cross sectional images. ${ }^{1217}$

\section{OVERRIDING OF THE GREAT ARTERIES}

There is a fundamental anomaly in septation in hearts with defects opening into both ventricular outlets. Overriding of a great artery is an important feature of some of these defects. The anomaly in these hearts should not be thought of as a simple hole in the ventricular septum. The leaflets of the overriding arterial valve form the roof of a conical space, the floor of which is the crest of the trabecular septum.
This space has both right and left ventricular margins. The right ventricular margin corresponds to the defect in similar hearts without an overriding arterial valve. The left ventricular margin is commonly called the "defect" in hearts with double outlet of the right ventricle. ${ }^{10}$ In these hearts, the ventricular septal defect should be considered as a three dimensional structure. All borders of the defect need to be described. ${ }^{11}$ Only cross sectional imaging permits this.

Overriding of either great arterial valve is a relatively common feature of all defects opening between both ventricular outlets, be they perimembranous, muscular, or doubly committed and juxtaarterial. When present, it is a marker for outlet extension of a defect. The morphology of these defects is excellently demonstrated in coronal sections through the right ventricle, ${ }^{18}$ but in such sections the extent of the override cannot be assessed. This is best done using long axis sections of the heart. Override of both great arteries can occur in the presence of a doubly committed defect. ${ }^{19}$

RELATION OF DEFECTS TO ARTERIAL VALVES The terms "subaortic" and "subpulmonary" have been used to describe different aspects of the spatial relations between defects and the arterial valves. If this relation is described precisely, rather than classifying it in this way, ambiguity can be avoided. Hence, one type of "subaortic" defect can be described as a perimembranous defect immediately adjacent to the aortic valve, ${ }^{2}$ while another "subaortic" defect could be described as opening between the subarterial outlets with overriding of the aortic valve. ${ }^{15}$ In the context of double outlet from the right ventricle with bilateral infundibulum, the relation between the defect and the arterial valves cannot be predicted from the morphology of the defect. The classifications of "subaortic" and "subpulmonary" can be used to describe, in this context, a judgement by the observer about the feasibility of surgically connecting the defect to either arterial valve. ${ }^{10} \mathrm{~A}$ full description of the relation of the defect to the arterial valves, however, is likely to be of more value than an arbitrary classification.

STRADDLING AND OVERRIDING OF THE ATRIOVENTRICULAR VALVES

Straddling and overriding of the mitral valve is also a feature of some outlet defects. Usually it is seen where there is a discordant ventriculoarterial connection or, as in this series, double outlet right ventricle with an overriding pulmonary valve, the so-called Taussig-Bing heart. ${ }^{120}$ Straddling and overriding of the tricuspid valve is a feature of a particular perimembranous inlet defect. It is associated with 8 
considerable malalignment of the atrial septum relative to the muscular ventricular septum, which no longer extends to the crux. Identification of this defect is straightforward because of the septal malalignment. These defects are the only exception to the rule that, in hearts with concordant atrioventricular connection, the conduction axis is always posterioinferior to perimembranous defects. ${ }^{20}$ Recognition of this anatomy is essential.

\section{CONCLUSION}

There is no need for a further classification of ventricular septal defects on morphological criteria and we have not attempted to produce one. Authors of the various existing classifications have chosen to emphasise different morphological features of the defects they studied, and they have, therefore, arrived at seemingly contradictory conclusions. We have not made a judgement that any one aspect of the morphology of ventricular septal defects is fundamental to their description. Ideally, all the morphological features need to be identified and described. We believe that, using the criteria we have described, it is possible virtually to achieve this aim. If, in this way, the morphology of a defect is completely described, its place in any of the diverse schemes of classification should be readily apparent. An additional problem is that some of the terms that have been used to describe the morphological features of ventricular septal defects are ambiguous, having been used by different workers to describe different features. To be of value descriptions need to be precise and so we have used terminology which describes the morphology of defects exactly and have discarded ambiguous terms. The great strength of cross sectional imaging is that it defines morphological detail at the margins of defects exceptionally well and, so, it is without equal in the clinical study of ventricular septal defects.

This work was performed when RHA was on a sabbatical from the Cardiothoracic Institute, Brompton Hospital, London SW3 6HP. It was supported by a grant from the Patrick Dick Memorial Fund.

\section{References}

1 Sutherland GR, Godman MJ, Smallhorn JF, Guiterras $P$, Anderson $R H$, Hunter $S$. Ventricular septal defects. Two dimensional echocardiographic and morphological correlations. Br Heart J 1982;47:31628.

2 Capelli H, Andrade JL, Somerville J. Classification of the site of ventricular septal defect by 2-dimensional echocardiography. Am J Cardiol 1983;51:1474-80.

3 Piot JD, Lucet P, Losay J, et al. Diagnosis and localisation of ventricular septal defects by two dimensional echocardiography. Arch Mal Coeur 1981;74:1001-9.
4 Anderson RH, Lenox CC, Zuberbuhler JR. Mechanisms of closure of perimembranous ventricular septal defects. Am J Cardiol 1983;52:3415.

5 Anderson RH, Lenox CC, Zuberbuhler JR. Morphology of ventricular septal defect associated with coarctation of the aorta. Br Heart J 1983;50:17681.

6 Anderson RH, Allwork SP, Ho SY, Lenox CC, Zuberbuhler JR. Surgical anatomy of the tetralogy of Fallot. J Thorac Cardiovasc Surg 1981;81:887-96.

7 Soto B, Becker AE, Moulaert AJ, Lie JT, Anderson RH. Classification of ventricular septal defects. $\mathrm{Br}$ Heart $J$ 1980;43:332-43.

8 Capelli H, Somerville J. Atypical Fallot's tetralogy with doubly committed sub-arterial defect. Diagnostic value of 2-dimensional echocardiography. Am J Cardiol 1983;51:282-5.

9 Silverman NH, Hunter S, Anderson RH, Ho SY, Sutherland GR, Davies MJ. Anatomical basis of cross sectional echocardiography. Br Heart J 1983;50: 421-31.

10 Macartney FJ, Rigby ML, Anderson RH, Stark J, Silverman NH. Double outlet right ventricle. Cross sectional echocardiographic findings, their anatomical explanation, and surgical relevance. $\mathrm{Br}$ Heart $J$ 1984;52:164-77.

11 Anderson RH, Becker AE, Tynan M. Description of ventricular septal defects-or how long is a piece of string? Int J Cardiol 1986;13:267-78.

12 Bierman FZ, Fellows K, Williams RG. Prospective identification of ventricular septal defects in infancy using subxiphoid two-dimensional echocardiography. Circulation 1980;62:807-17.

13 Hagler DJ, Edwards WD, Seward JB, Tajik AJ. Standardized nomenclature of the ventricular septum and ventricular septal defects, with applications for twodimensional echocardiography. Mayo Clin Proc 1985;60:741-52.

14 Milo S, Ho SY, Wilkinson JL, Anderson RH. Surgical anatomy and atrioventricular conduction tissues of hearts with isolated ventricular septal defects. $J$ Thorac Cardiovasc Surg 1980;79:244-55.

15 Lev M, Bharati S, Memg CCL, Liberthson RR, Paul MH, Idriss F. A concept of double outlet right ventricle. J Thorac Cardiovasc Surg 1972;64:271-81.

16 Ando M, Takao A. Pathological anatomy of ventricular septal defect associated with aortic valve prolapse and regurgitation. Heart and Vessels 1986;2:117-26.

17 Cheatham JP, Latson LA, Gutgesell HP. Ventricular septal defect in infancy: detection with two-dimensional echocardiography. Am J Cardiol 1981;47:85-9.

18 Magherini A, Margiotta C, Simonetti L, et al. Crosssectional echocardiography with pulsed and continuous wave Doppler in management of ventricular septal defects. Int J Cardiol 1987;15:317-28.

19 Ueda M, Becker AE. Double outlet right ventricle: an unusual variant with overriding of both great arteries, absent outlet septum and mitral-to-aortic-to-pulmonary continuity. Int J Cardiol 1986;12:155-60.

20 Milo S, Ho SY, Macartney FJ, et al. Straddling and overriding atrioventricular valves; morphology and classification. Am J Cardiol 1979;44:1122-34. 